\title{
Ein Fall von Aluminose der Atemwege mit wahrscheinlich sekundärer pleuroparenchymaler Fibroelastose
}

\author{
Yuki Yabuuchi $^{\mathrm{a}}$ Hitomi Goto ${ }^{\mathrm{a}}$ Mizu Nonaka ${ }^{\mathrm{a}}$ Hiroaki Tachi ${ }^{\mathrm{a}}$ Tatsuya Akiyama ${ }^{\mathrm{a}}$ \\ Naoki Arai ${ }^{a}$ Hiroaki Ishikawa ${ }^{a}$ Kentaro Hyodo $^{a}$ Kenji Nemoto ${ }^{a}$ Yukiko Miura $^{a}$ \\ Isano Hase $^{a}$ Shuji Oh-ishi ${ }^{a}$ Kenji Hayashihara ${ }^{a}$ Takefumi Saito ${ }^{a}$ Tatsuya Chonan ${ }^{b}$ \\ ${ }^{a}$ Department of Respiratory Medicine, National Hospital Organization, Ibaraki Higashi National Hospital, Ibaraki, Japan; \\ ${ }^{b}$ Department of Medicine, Nikko Memorial Hospital, Ibaraki, Japan
}

Schlüsselwörter

Interstitielle Pneumonie · Berufsbedingte Lungenerkrankung ·

Transbronchiale Lungenbiopsie · Elementaranalyse

\section{Zusammenfassung}

Hintergrund: Die exzessive Inhalation von Aluminiumstaub führt gelegentlich zu einer überwiegend die Oberlappen betreffenden Lungenfibrose, die einer idiopathischen pleuroparenchymalen Fibroelastose (IPPFE) ähnelt und bei der es sich den Annahmen zufolge um eine sekundäre PPFE handelt.

Fallbericht: Ein 67-jähriger Mann, der 50 Jahre lang in einem aluminiumverarbeitenden Unternehmen gearbeitet hatte, stellte sich mit Belastungsdyspnoe in unserer Klinik vor. Die Computertomographie (CT) des Thorax zeigte eine bilaterale dichte subpleurale Konsolidierung in den oberen und mittleren Lungenfeldern entsprechend einer IPPFE; eine etwaige sekundäre PPFE im Zusammenhang mit einer Aluminose wurde jedoch nicht ausgeschlossen. Aufgrund des kritischen Zustands des Patienten erfolgten eine transbronchiale Lungenbiopsie (TBLB) anstatt einer chirurgischen Lungenbiopsie sowie eine Elementaranalyse der entnommenen
Gewebeprobe. Da die mittels TBLB gewonnene Probe bedauerlicherweise kein Alveolargewebe enthielt, konnte die Diagnose PPFE nicht pathologisch gesichert werden. Die radiologischen Befunde sprachen jedoch stark für eine PPFE. In der Elementaranalyse wurden hohe Mengen an Aluminium in den Bronchiolarwänden nachgewiesen, so dass die Diagnose einer Aluminose der Atemwege mit wahrscheinlich sekundärer PPFE infolge Aluminiumexposition gestellt wurde.

Schlussfolgerungen: Eine TBLB mit Elementaranalyse kann zur Abgrenzung der idiopathischen PPFE von sekundären Ursachen bei staubbedingten Erkrankungen wie Aluminose hilfreich sein. Der vorliegende Fall zeigt, dass die Inhalation von Aluminium eine sekundäre PPFE verursachen kann; ein besonderes Augenmerk sollte auf die Vermeidung einer weiteren Exposition gelegt werden.

\section{Hintergrund}

Die pleuroparenchymale Fibroelastose (PPFE) ist radiologisch durch eine überwiegend die Oberlappen betreffende und pathologisch nicht klassifizierte interstitielle Pneumonie gekennzeichnet [1]. Die meisten PPFE-Fälle wurden als idiopathisch einge- stuft. Gelegentlich tritt die Erkrankung jedoch als Folge anderer Umstände auf, wie Medikamentengabe, Infektionen, nach einer Transplantation und Staubexposition [2]. Insbesondere liegen Berichte über PPFE-ähnliche Läsionen im Zusammenhang mit Exposition gegenüber Stäuben wie Asbest, Aluminium und Aluminiumsilikat vor $[3,4]$.

\section{KARGER}

Fax +497614520714 information@karger.com www.karger.com

Accessible online at: www.karger.com/kkp

Karger 2019 Die Autoren
Open access

This article is licensed under the Creative Commons Attribution 4.0 International License (CC BY) (http://www.karger.com/Services/Open AccessLicense). Usage, derivative works and distribution are permitted provided that proper credit is given to the au-
thor and the original publisher.
Yuki Yabuuchi

Department of Respiratory Medicine, Notional Hospital Organization

Ibaraki Higashi National Hospital

325 Terunuma, Tokai, Naha District, Ibaraki 319-1113, Japan

jabberflag@ hotmail.co.jp 
In der vorliegenden Arbeit beschreiben wird den Fall eines Arbeiters mit Aluminiumstaub-Exposition und mutmaßlicher sekundärer PPFE, die mittels Computertomographie (CT) des Thorax, transbronchialer Lungenbiopsie (TBLB) und Elementaranalyse der entnommenen Gewebeprobe diagnostiziert wurde. Der Bericht liefert neue Erkenntnisse über die sekundäre sowie die idiopathische PPFE.

\section{Fallbericht}

Bei dem Patienten handelte es sich um einen 67-jährigen männlichen Nichtraucher mit unauffälliger medizinischer Vorgeschichte. Er wurde wegen einer seit 3 Monaten bestehenden, zunehmenden Dyspnoe in unsere Klinik aufgenommen. Seine Eltern führten einen Produktionsbetrieb in einer Kleinstadt und er hatte seit seinem 18. Lebensjahr in der Aluminium- und Messingverarbeitung zur Herstellung von Kamerateilen gearbeitet. Wegen seines stark eingeschränkten Sehvermögens arbeitete er sehr nah an der Schneidemaschine (Abb. 1) und trug dabei keine Staubschutzmaske.

Bei der Aufnahme war der Patient bei vollem Bewusstsein, die Körpergröße betrug $160 \mathrm{~cm}$ und das Körpergewicht $47 \mathrm{~kg}$; die Körpertemperatur lag bei $36,8{ }^{\circ} \mathrm{C}$ und die perkutan gemessene Sauerstoffsättigung $\left(\mathrm{SpO}_{2}\right)$ bei $94 \%$ (Raumluft). Auskultatorisch war, insbesondere über beiden oberen Lungenabschnitten, ein leises Knisterrasseln zu hören. Ansonsten fanden sich in der körperlichen Untersuchung keine pathologischen Befunde. Die Laborergebnisse zeigten erhöhte Serumspiegel von KL-6 (Krebs von den Lungen-6; $1.631 \mathrm{U} / \mathrm{ml}$ ) und SP-D (Surfactant-Protein D; 157,3 $\mathrm{ng} / \mathrm{ml}$ ) im Vergleich zu den Normwerten von $<500 \mathrm{U} / \mathrm{ml}$ bzw. $<110 \mathrm{ng} / \mathrm{ml}$ ). Die antinukleären und autologen Antikörper waren alle negativ. In den Thorax-Röntgenaufnahmen waren lineare und retikulonoduläre Verdichtungen mit deutlicher bilateraler Pleuraverdickung in den oberen Lungenfeldern erkennbar (Abb. 2). Die CT-Bilder des Thorax zeigten eine bilaterale dichte subpleurale Konsolidierung, Bronchiektasen und Milchglastrübungen in beiden Oberlappen (Abb. 3). Die Lungenfunktionsuntersuchungen ergaben eine restriktive Ventilationsstörung: Vitalkapazität (vital capacity, VC) von 1,12 l (34,6\% des Sollwerts), forciertes expiratorisches Volumen in 1 Sekunde $\left(\mathrm{FEV}_{1}\right)$ von 0,991 (42,5\% des Sollwerts), FEV 1 /FVC (forcierte Vitalkapazität) von $74,4 \%$ und totale Lungenkapazität (total lung capacity, TLC) von 2,48 1 (47,7\% des Sollwerts). Der Patient war nicht in der Lage, den Diffusionstest korrekt auszuführen. Es erfolgte eine Bronchoskopie mit transbronchialer Lungenbiopsie, bei der Gewebeproben aus dem rechten Oberlappen entnommen wurden (B2). Die Untersuchung der bronchoalveolären Lavage-Flüssigkeit, die durch Einbringung von $150 \mathrm{ml}$ Kochsalzlösung gewonnen wurde, ergab eine im Normbereich liegende Gesamtzellzahl von $6,0 \times 104 / \mathrm{ml}$ mit 3\% Lymphozyten, $1 \%$ Neutrophile, 2\% Eosinophile und $94 \%$ Makrophagen (Cluster of Differentiation CD 4/8-Verhältnis von $2,31)$. In den entnommenen Gewebeproben fanden sich Fragmente der Bronchiolarwände mit lymphozytärer Infiltration der interstitiellen Räume; bedauerlicherweise enthielten sie jedoch kein
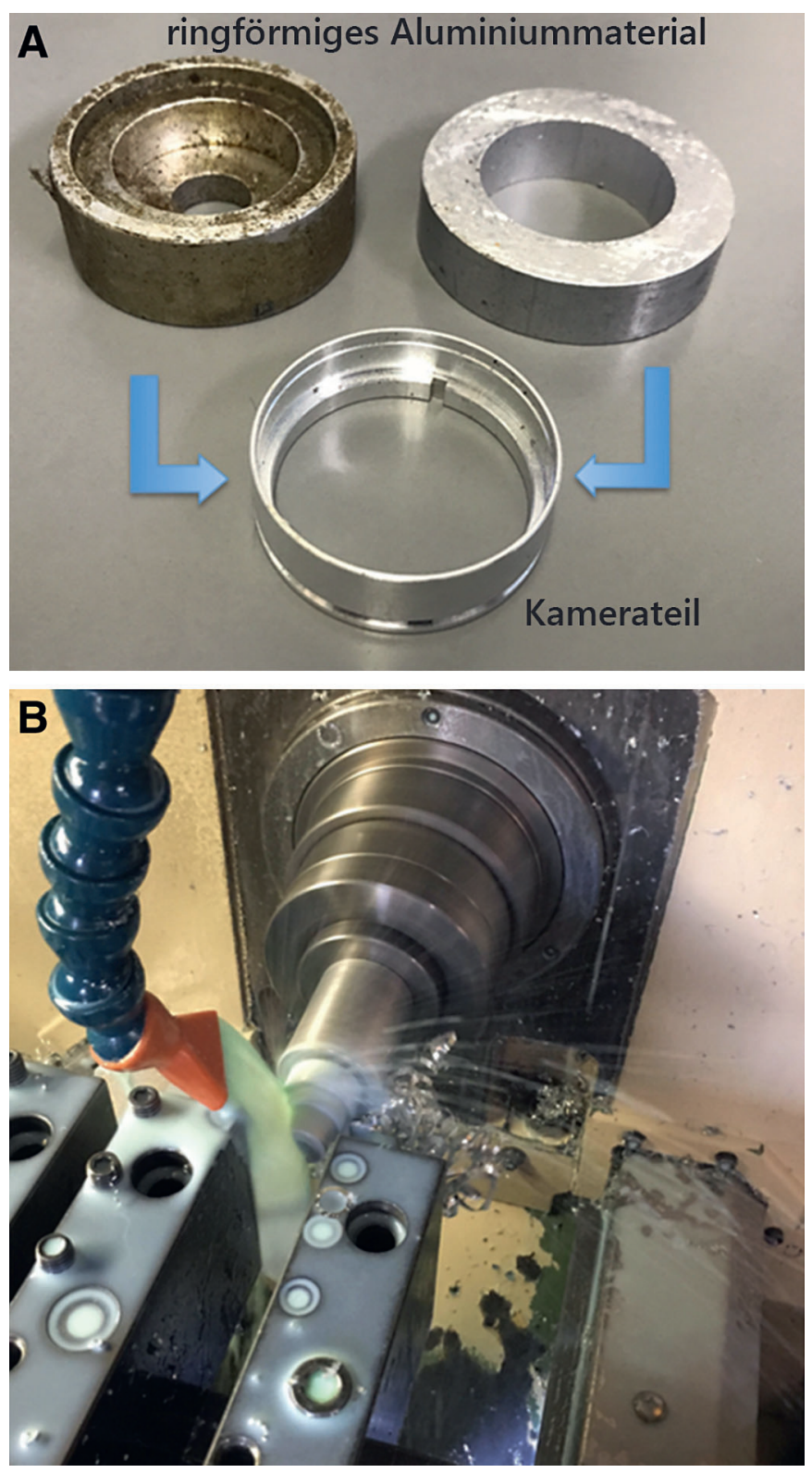

Abb. 1. Die Arbeit des Patienten bestand im Fräsen von ringförmigem Aluminiummaterial zur Herstellung von Kamerateilen (siehe die beiden Abbildungen im oberen Bild bzw. die Abbildung darunter) (a) mit einer Drehmaschine (b). Dabei ist die Aufbringung von Schneidöl auf das Material vorgeschrieben (b), was unser Patient jedoch versäumte, so dass er vermutlich eine größere Menge aluminiumhaltigen Rauchs eingeatmet hat als andere Arbeiter mit der gleichen Art von Arbeit.

Alveolargewebe. Wegen der kritischen respiratorischen Erkrankung des Patienten wurde kein diagnostischer chirurgischer Eingriff durchgeführt. Stattdessen erfolgte eine Elementaranalyse an der mittels TBLB gewonnenen Gewebeprobe und es wurde der Gehalt an Aluminium und anderen Partikeln in der TBLB-Gewebeprobe bestimmt.

Dabei fand sich in der Gewebeprobe eine relativ hohe Menge an Aluminium, wohingegen andere Elemente, darunter Eisen und Siliziumdioxid, nur in Spuren nachweisbar waren (Abb. 4). Aufgrund der Ergebnisse der histologischen Untersuchung sowie der 


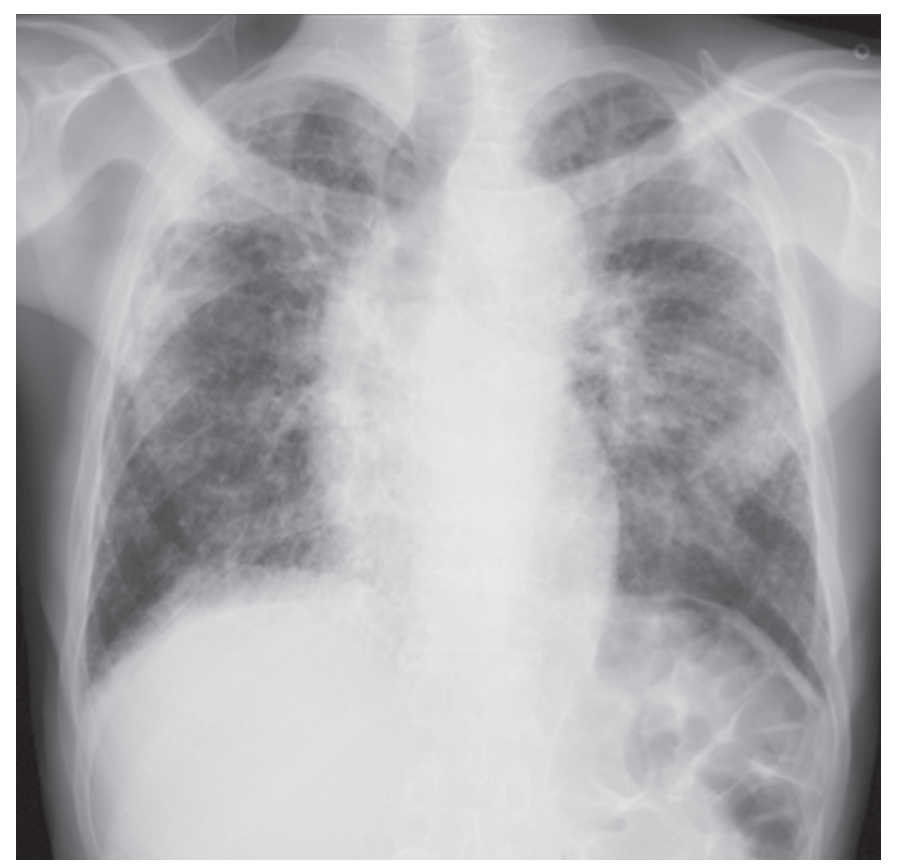

Abb. 2. Die Röntgenaufnahme des Thorax zeigt eine bilaterale Pleuraverdickung in den oberen und mittleren Lungenfeldern. Das verringerte Lungenvolumen und die retikulonodulären Verdichtungen, die sich von subpleural bis tief in die Lunge erstreckten, sprachen für eine Lungenfibrose. Durch den Zug der beiden Oberlappen ist die Trachealbifurkation aufgespreizt.

Elementaranalyse und der Berufsanamnese wurde eine Aluminose der Atemwege diagnostiziert. Der Zustand des Patienten verschlechterte sich zunehmend, so dass er eine Langzeit-Sauerstofftherapie für zu Hause und den antifibrotischen Wirkstoff Pirfenidon erhielt. Sechs Monate später trat ein Pneumothorax auf und es wurde eine Thoraxdrainage gelegt. Dennoch verschlechterte sich sein Zustand weiter und er verstarb einen Monat später infolge eines hyperkapnischen respiratorischen Versagens. Seine Angehörigen verweigerten die Zustimmung zu einer Autopsie.

\section{Diskussion}

Die Ätiologie der pleuroparenchymalen Fibroelastose ist bislang nicht bekannt, doch wurde die Inhalation von Staub als eine mögliche Ursache der sekundären PPFE diskutiert [3,4]. Definitionsgemäß handelt es sich bei der PPFE um eine überwiegend die Oberlappen betreffende subpleurale Fibrose, deren Diagnose radiologisch und pathologisch zu sichern ist $[1,4]$.

In unserem Fall enthielten die entnommenen Proben nur Bronchiolarwandfragmente und kein Alveolargewebe, so dass ein histologischer Nachweis der PPFE nicht möglich war. Die CT-Bilder des Thorax zeigten eine überwiegend die Oberlappen betreffende Pleuraverdickung mit Lungenfibrose, Bronchiektasen und Schrumpfung der Lunge, entsprechend den radiologischen Zeichen einer PPFE $[1,2]$. Andererseits fanden sich Zeichen, die nicht $\mathrm{zu}$ einer PPFE passten, wie etwa das Vorliegen einer sowohl die
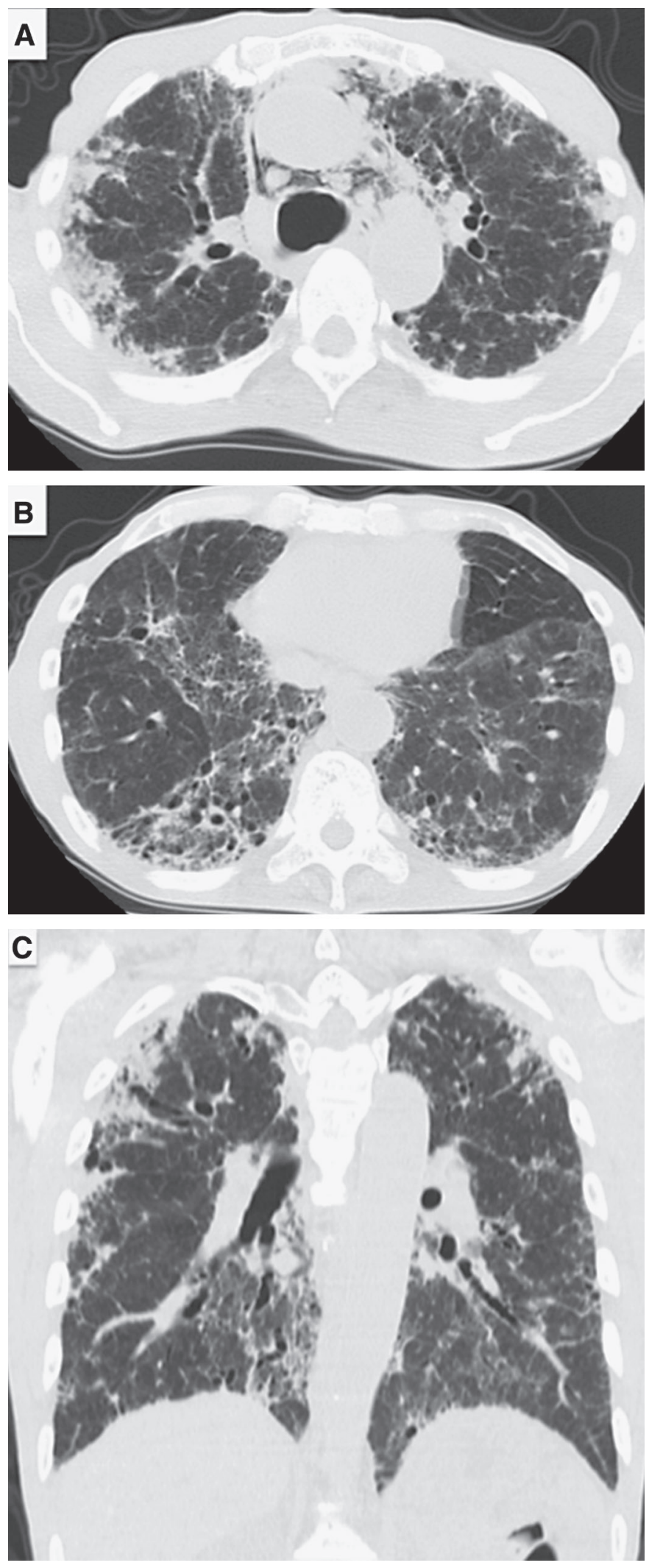

Abb. 3. Die hochauflösende Computertomographie (high-resolution computed tomography, HRCT) zeigt ausgeprägte bilaterale Pleuraverdickungen mit Schrumpfung und Verziehung der Oberlappen (a, c). Außerdem bestand ein Mediastinalemphysem (a) und die retikulonodulären Verdichtungen und Bronchiektasien erstreckten sich bis weit in die unteren Lungenabschnitte $(\mathbf{b}, \mathbf{c})$. 


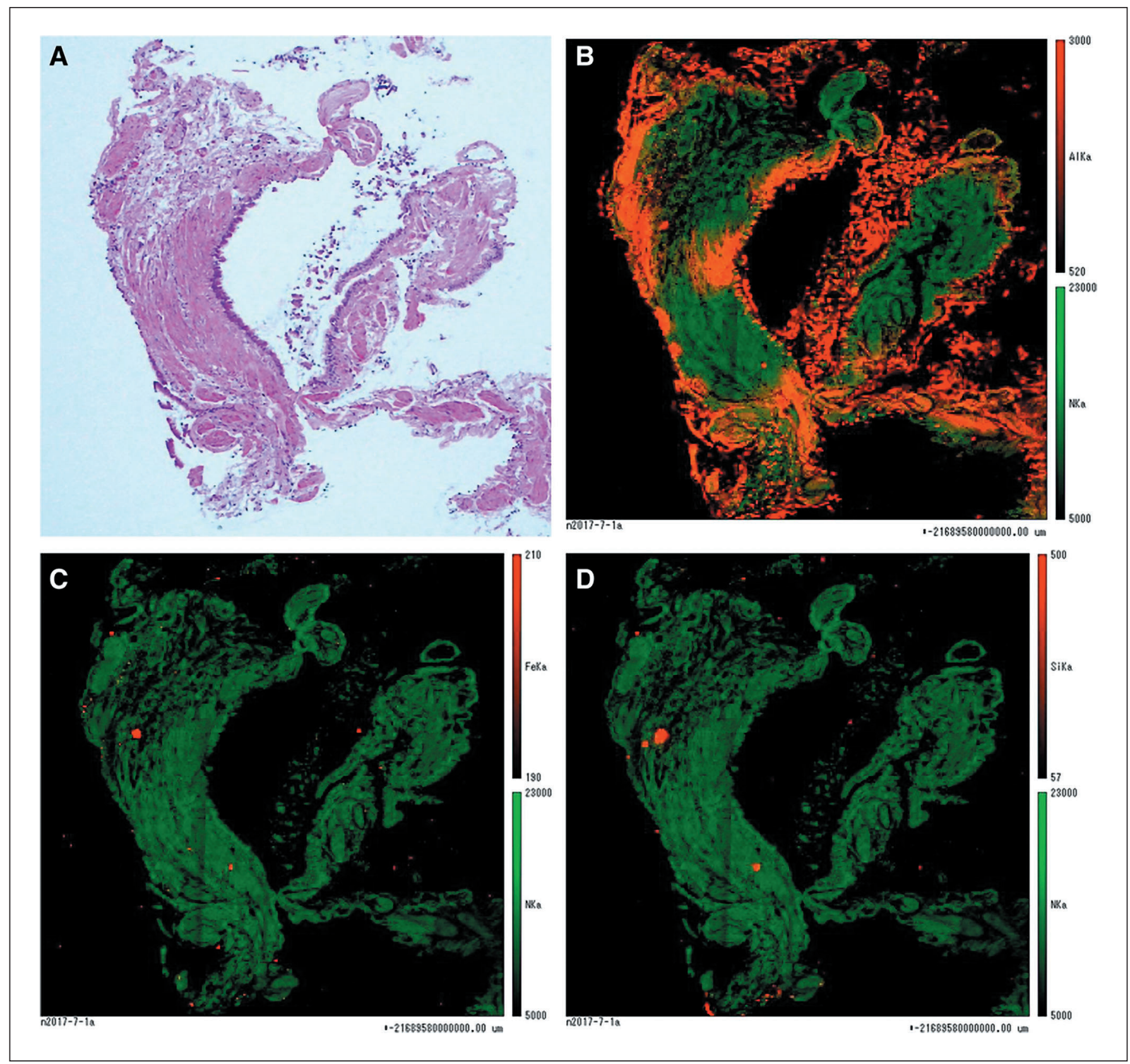

Abb. 4. Elementaranalyse der Gewebeproben mittels Elektronenstrahlmikroanalyse (ESMA). In einem durch TBLB (transbronchiale Lungenbiopsie) gewonnenen Bronchialwandfragment wurde nach Färbung mit Hämatoxylin-Eosin Aluminium nachgewiesen (a). Die Ablagerung der Elemente in der Gewebeprobe ist durch die rote bis gelbe Farbkennzeichnung dargestellt. Grün zeigt die Ablagerung von Stickstoff an, der als Kontrolle verwendet wurde. In der ElektronenstrahImikroanalyse fanden sich erhebliche Mengen an Aluminium, erkennbar an der roten bis gelben Farbe (b), wohingegen Eisen und Siliziumdioxid in deutlich geringeren Mengen als Aluminium nachgewiesen wurden (c, d).

Unter- als auch die Oberlappen betreffenden Fibrose, und neben der subpleuralen Fibrose wurden tief in der Lunge gelegene Läsionen beobachtet. Zudem ist bei PPFE in den CT-Aufnahmen des Thorax typischerweise eine klare Demarkation zwischen abnormen und normalen Lungenabschnitten erkennbar [5], wohingegen diese Abgrenzung im vorliegenden Fall nicht eindeutig möglich war. Ob es sich bei den retikulonodulären Verdickungen in den tiefen Lungenabschnitten um ein Element der PPFE handelt, ist strittig.
Die durch chronische Inhalation von aluminiumhaltigem Staub oder Rauch verursachte Lungenfibrose wird als Aluminose bezeichnet [6]. Radiologisch ist sie gewöhnlich durch bilaterale, hauptsächlich die oberen Lungenabschnitte betreffende, retikulonoduläre oder irreguläre Verdichtungen mit Pleuraverdickung gekennzeichnet, die nur in seltenen Ausnahmefällen überwiegend in den unteren Lungenabschnitten auftreten, wie dies gelegentlich bei PPFE-ähnlichen Läsionen der Fall ist [4, 7]. Histologisch ist die Aluminose durch eine subpleurale Fibrose mit em- 
physematösen Läsionen infolge fibrotischer Vernarbung gekennzeichnet [8]. Für die Diagnose einer Aluminose der Lunge ist der Nachweis erhöhter Aluminiumkonzentrationen im Alveolargewebe erforderlich $[8,9]$.

Im unserem Fall sprachen die erhöhten Serumspiegel der Biomarker KL- 6 und SP-D sowie die restriktive Lungenfunktionsstörung und die radiologischen Befunde für das Vorliegen einer schweren Lungenfibrose. Wir entschieden, lediglich eine Elementaranalyse der mittels TBLB gewonnenen Bronchiolarwandfragmente durchzuführen, da ein invasiver Eingriff wie beispielsweise eine chirurgische Lungenbiopsie (surgical lung biopsy, SLB) den Zustand des Patienten möglicherweise weiter verschlechtert hätte. Die Elementaranalyse ergab ausgeprägte Aluminiumablagerungen mit geringen Mengen an Eisen und Siliziumdioxid, entsprechend der Definition einer Aluminose der Lunge. Das Ergebnis zeigte, dass es zu einer Inhalation großer Mengen an Aluminium gekommen war, die möglicherweise zur Entstehung der PPFEähnlichen Läsionen geführt hat.

Die pleuroparenchymale Fibroelastose hat eine schlechte Prognose und es gibt bisher keine wirksamen Therapien. Wir verabreichten den antifibrotischen Wirkstoff Pirfenidon, der zur Behandlung der idiopathischen pulmonalen Fibrose (IPF) eingesetzt wird. Es liegen keine Belege für die Wirksamkeit von Pirfenidon bei anderen fibrotischen Lungenerkrankungen außer IPF vor; eine Studie, in der die Wirksamkeit beurteilt wird, läuft jedoch derzeit. In unserem Fall hatte sich die Dyspnoe 6 Monate nach Therapiebeginn mit Pirfenidon nicht gebessert und es kam zu einem Pneumothorax, in dessen Folge der Patient verstarb.

Wie im vorliegenden Fall sollte bei Auftreten sekundärer PPFEähnlicher Läsionen im Zusammenhang mit Staubexposition eine transbronchiale Lungenbiopsie erwogen werden. Patienten, bei denen eine sekundäre PPFE infolge Staubexposition diagnostiziert wird, können die Befunde und Ergebnisse unter Umständen verwenden, um eine finanzielle Entschädigung zu erhalten.

\section{Schlussfolgerung}

Die vorliegende Kasuistik beschreibt den Fall einer überwiegend die Oberlappen betreffenden Lungenfibrose bei einem Aluminiumschweißer. Sie zeigt, dass die übermäßige Inhalation von Aluminium eine sekundäre PPFE verursachen kann.

\section{Danksagung}

Wir danken Hiroshi Moriyama, MD, vom Department of Homeostatic Regulation and Development, Niigata University Graduate School of Medical Dental Sciences, Niigata, Japan, für die Durchführung der Elementaranalyse.

\section{Finanzielle Unterstützung}

Nicht zutreffend.

\section{Verfügbarkeit der Daten und Materialien}

Nicht zutreffend.

\section{Autorenbeiträge}

Alle Autoren haben die finale Fassung des Manuskripts gelesen und freigegeben.

\section{Genehmigung durch die Ethikkommission und Einwilli- gung zur Teilnahme}

Die Studie wurde von der Ethikkommission des Ibaraki Higassi National Hospital, Japan, genehmigt.

\section{Zustimmung zur Veröffentlichung}

Von den Angehörigen des Patienten wurde nach Aufklärung eine schriftliche Einverständniserklärung für die Veröffentlichung des vorliegenden Fallberichts und der dazugehörigen Bilder eingeholt. Der Wortlaut der schriftlichen Einverständniserklärung ist von der Redaktion dieser Zeitschrift zur Überprüfung erhältlich.

\section{Interessenskonflikte}

Die Autoren erklären, dass keine Interessenskonflikte bestehen.

\section{Lizenzangabe}

Yuki Yabuuchi, Hitomi Goto, Mizu Nonaka, Hiroaki Tachi, Tatsuya Akiyama, Naoki Arai, Hiroaki Ishikawa, Kentaro Hyodo, Kenji Nemoto, Yukiko Miura, Isano Hase, Shuji Oh-ishi, Kenji Hayashihara, Takefumi Saito, Tatsuya Chonan:A case of airway aluminosis with likely secondary pleuroparenchymal fibroelastosis, Multidiscip Respir Med 2019;14:15 (https:// doi.org/10.1186/s40248-019-0177-4). ${ }^{\odot}$ The Author(s) 2019 (Übersetzung, Publisher's note gekürzt), lizensiert unter CC BY 4.0 (https://creativecommons.org/licenses/by/4.0/deed.de).

\section{Literatur}

1 Travis WD, Costabel U, Hansell DM, et al.: An official American Thoracic Society/European Respiratory Society statement: update of the international multidisciplinary classification of the idiopathic interstitial pneumonias. Am J Respir Crit Care Med 2013;188:733-748.

$\checkmark 2$ von der Thüsen JH. Pleuroparenchymal fibroelastosis: its pathological characteristics. Curr Respir Med Rev 2013;9:238-247.

$>$ Huang Z, Li S, Zhu Y, et al.: Pleuroparenchymal fibroelastosis associated with aluminosilicate dust: a case report. Int J Clin Exp Pathol 2015;8:8676-8679.
4 Watanabe K: Pleuroparenchymal fibroelastosis: its clinical characteristics. Curr Respir Med Rev. 2013;9:229-237.

5 Bonifazi M, Montero MA, Renzoni EA: Idiopathic pleuroparenchymal fibroelastosis. Curr Pulmonol Rep 2017;6:9-15.

6 Chino H, Hagiwara E, Sugisaki M, et al. : Pulmonary aluminosis diagnosed with in-air microparticle induced X-ray emission analysis of particles. Intern Med 2015;54:2035-2040.
Smolkova P, Nakladalova M: The etiology of occupational pulmonary aluminosis - the past and the present. Biomed Pap Med Fac Univ Palacky Olomouc Czech Repub 2014;158: 535-538.

8 Kraus T, Schaller KH, Angerer J, et al.: Aluminosis - detection of an almost forgotten disease with HRCT. J Occup Med Toxicol 2006;1: 4.

9 Yasui M: Aluminum lung. Jpn Chest Dis 2011; 70:1249-1258. 